Research Article

\title{
Phyloproteomic Analysis of 11780 Six-Residue-Long Motifs Occurrences
}

\author{
O. V. Galzitskaya and M. Yu. Lobanov \\ Institute of Protein Research, Russian Academy of Sciences, 4 Institutskaya Street, Pushchino, Moscow Region 142290, Russia
}

Correspondence should be addressed to O. V. Galzitskaya; ogalzit@vega.protres.ru

Received 11 July 2014; Accepted 3 November 2014

Academic Editor: Peter F. Stadler

Copyright (C) 2015 O. V. Galzitskaya and M. Yu. Lobanov. This is an open access article distributed under the Creative Commons Attribution License, which permits unrestricted use, distribution, and reproduction in any medium, provided the original work is properly cited.

\begin{abstract}
How is it possible to find good traits for phylogenetic reconstructions? Here, we present a new phyloproteomic criterion that is an occurrence of simple motifs which can be imprints of evolution history. We studied the occurrences of 11780 six-residue-long motifs consisting of two randomly located amino acids in 97 eukaryotic and 25 bacterial proteomes. For all eukaryotic proteomes, with the exception of the Amoebozoa, Stramenopiles, and Diplomonadida kingdoms, the number of proteins containing the motifs from the first group (one of the two amino acids occurs once at the terminal position) made about 20\%; in the case of motifs from the second (one of two amino acids occurs one time within the pattern) and third (the two amino acids occur randomly) groups, $30 \%$ and 50\%, respectively. For bacterial proteomes, this relationship was 10\%, 27\%, and 63\%, respectively. The matrices of correlation coefficients between numbers of proteins where a motif from the set of 11780 motifs appears at least once in 9 kingdoms and 5 phyla of bacteria were calculated. Among the correlation coefficients for eukaryotic proteomes, the correlation between the animal and fungi kingdoms (0.62) is higher than between fungi and plants (0.54). Our study provides support that animals and fungi are sibling kingdoms. Comparison of the frequencies of six-residue-long motifs in different proteomes allows obtaining phylogenetic relationships based on similarities between these frequencies: the Diplomonadida kingdoms are more close to Bacteria than to Eukaryota; Stramenopiles and Amoebozoa are more close to each other than to other kingdoms of Eukaryota.
\end{abstract}

\section{Introduction}

By the middle of the XXth century, it had become clear that all living organisms of cellular texture are divided into two groups or kingdoms, prokaryotes and eukaryotes, according to structural peculiarities of their cells. It was long believed that the terms "prokaryotes" and "bacteria" are synonyms for the same independent evolutionary branch of living organisms. However, about 30 years ago, molecular comparisons of base sequences of ribosomal RNAs provided grounds to divide prokaryotes into at least two independent branches, Eubacteria and Archaebacteria, which differ in their origin [1]. Later, these data were generalized and the term DOMAIN was suggested, which is the branch that has the highest rank in the hierarchic taxonomy [2]. These DOMAINS are Bacteria, Archaea, and Eukaryota.

Protein phylogeny was developed simultaneously with RNA phylogeny $[3,4]$. Protein phylogeny is similar to the developed RNA phylogeny because it is based on the division of living organisms into three DOMAINS. RNA and protein phylogenies are based on the alignments of sequences from different organisms, and most phylogenetic methods are based on comparison of protein or nucleic acid sequences in their aligned parts. The conventional tree-building methods for phylogenetic reconstructions are neighbor joining (NJ) [5], maximum parsimony (MP) [6], and maximum likelihood (ML) [7]. Moreover, there is an additional approach as alignment-free phylogeny methods based on k-mer appearance in genomic DNA [8-12].

The understanding of how different major groups of organisms are related to each other and the tracing of their evolution from the common ancestor remains controversial and unsolved. In recent years, the wealth of new information based on a large number of gene and protein sequences has become available. At present, a phylogenetic analysis can be carried out based on either nucleic acid or protein sequences. 
Nonetheless, the phylogenetic relationship among the kingdoms Animalia, Plantae, and Fungi remains uncertain despite extensive attempts to clarify it. The first hypothesis states that Animalia is more closely related to Plantae [13-15]. The second one supports Plantae and Fungi grouping [16]; the third one, Animalia and Fungi [17-23]. To elucidate evolutionary relationships among different proteomes we will consider the occurrence of some simple motifs which can be imprints of evolution history.

What candidates can be stated as simple motifs? We have done several investigations in this direction. First, by combining the motif discovery and disorder protein segment identification in the Protein Data Bank (PDB: http://www .rcsb.org/), we have compiled the largest database of disordered patterns (171) from the clustered PDB where identity between chains inside a cluster is larger than or equal to $75 \%$ using simple rules of selection [21-24]. Second, among these patterns, the patterns with low complexity are more abundant and the length of these motifs is six residues. Third, the patterns with frequent occurrence in proteomes have low complexity (PPPPP, GGGGG, EEEED, HHHH, KKKKK, SSTSS, and QQQQQP), and the type of patterns varies across different proteomes [21]. It is supposed that if an amino acid motif possesses no definite spatial structure in most protein structures, it is likely to be disordered in a protein with an unknown spatial structure [21]. Therefore, the patterns with the length of six residues and low complexity, which are, for example, homorepeats of 20 amino acids, are the major candidates for this role. The length of six residues is important: (1) the experiments performed demonstrated that a minimum repeat size of 6 histidine residues was required for efficient protein translocation to nuclear speckles [25]; (2) six-residue patches affect the folding/aggregation features of proteins, and they are important "words" for the understanding of protein dynamics [26]; (3) nucleation sites are constrained by patches of approximately six residues [27, $28]$.

It has been found that homorepeats of some amino acids (runs of a single amino acid) occur more frequently than others and the type of homorepeats varies across different proteomes [21]. For example, EEEEEE appears to be the most frequent for all considered proteomes for Chordata, QQQQQQ for Arthropoda, and SSSSSS for Nematoda. A comparative analysis of the number of proteins containing 6-residue-long homorepeats and the 109 disordered selected patterns in 123 proteomes has demonstrated that the correlation coefficients between numbers of proteins are higher inside the considered kingdom than between them [21]. In these proteins a six-residue-long homorepeat occurs at least once for each of the 20 types of amino acid residues and 109 disordered patterns from the library appearing in 9 kingdoms of Eukaryota and 5 phyla of Bacteria.

Here, we present a new phyloproteomic criterion which is based on the peculiarities of amino acid sequences which is an occurrence of some simple motifs which can be imprints of evolution history. In this work, we focus our attention on studying the frequency of six simple amino acid motifs consisting of two randomly located amino acids (11780 motifs) in 122 eukaryotic and bacterial proteomes.

\section{Materials and Methods}

2.1. Construction of the Library of Six-Residue-Long Motifs . We constructed the library of all possible motifs composed of two amino acids, with the assumption that each amino acid could be at any position and at any ratio and that such a motif was six amino acids long [29]. There were $11780=\left(2^{6}-2\right) \cdot C_{20}^{2}$ such motifs in total (excluding two homorepeats for every amino acid pair). The obtained motifs could be divided into three groups. The first group contains the motifs where one of the two amino acids occurs only once and occupies the first or sixth (i.e., outside) position. The second group includes motifs where the second amino acid also occurs once but is inside the motif. The third group contains all the other motifs where each of the two amino acids occurs at least twice and in any order.

2.2. Database of Proteomes. We considered 3279 proteomes from the EBI site (ftp://ftp.ebi.ac.uk/pub/databases/SPproteomes/last_release/uniprot/proteomes/). A preliminary analysis showed that the number of proteins with at least one occurrence of homorepeats, 6 residues long, is less than 500 for proteomes with an overall number of residues below $2,500,000$. Even so, only 22 proteomes out of 3156 have more than 100 proteins with at least one occurrence of 6-residue homorepeats. These data provided grounds for our research involving only proteomes with an overall number of residues exceeding 2,500,000.

We obtained 122 proteomes taking into account the length of proteomes representing 9 kingdoms of eukaryotes and 5 phyla of Bacteria (see Table 1 in [21]). Unfortunately, only three kingdoms of eukaryotes (Metazoa, Viridiplantae, and Fungi) are given at http://www.ncbi.nlm.nih.gov/Taxonomy. In other cases, the rank of kingdom is missing. In such situations, we chose the highest taxonomic category following from the subkingdom of eukaryotes instead of the kingdom. We chose 97 out of 120 eukaryotic proteomes and a small number of bacterial proteomes. The smallest eukaryotic proteome belongs to Hemiselmis andersenii, class Cryptophyta. It is evident that 498 proteins with an overall number of 167,452 amino acid residues are not sufficient for reliable statistics. Historically, the superkingdom of Bacteria is divided into phyla but not kingdoms. We preferred to consider such phyla separately.

Among 97 eukaryotic proteomes, 17 belong to the kingdom of Metazoa or animals: Homo sapiens (51778 protein sequences), Bos taurus (18405), Mus musculus (42120), Rattus norvegicus (28166), Gallus gallus (12954), Danio rerio (21576), and Tetraodon nigroviridis (27836) belong to Chordata phylum; Drosophila melanogaster (15101), Drosophila pseudoobscura (16000), Aedes aegypti (16042), Anopheles darlingi (11437), and Anopheles gambiae (12455) to arthropods; Caenorhabditis briggsae (18531), Caenorhabditis elegans (23817), Loa loa (16271), and Trichinella spiralis (16040) to nematodes; Nematostella vectensis (24435) belongs to Cnidaria phylum.

2.3. Calculation of Correlation Coefficient. The vectors of 11780 values for each type of motif are compared between 
TABLE 1: 11780 motifs that frequently occur in 123 proteomes.

\begin{tabular}{|c|c|c|c|c|c|c|c|}
\hline \multicolumn{2}{|c|}{11780} & \multicolumn{2}{|c|}{ The first group } & \multicolumn{2}{|c|}{ The second group } & \multicolumn{2}{|c|}{ The third group } \\
\hline EEEEED & 6744 & EEEEED & 6744 & EDEEEE & 4248 & APAPAP & 3543 \\
\hline QQQQQP & 6300 & QQQQQP & 6300 & STSSSS & 4166 & DDEEEE & 3464 \\
\hline DEEEEE & 6165 & DEEEEE & 6165 & NNNNSN & 4030 & SGSGSG & 3423 \\
\hline TSSSSS & 6135 & TSSSSS & 6135 & EEEEDE & 3995 & PAPAPA & 3392 \\
\hline SGGGGG & 6117 & SGGGGG & 6117 & NSNNNN & 3992 & EEEEDD & 3292 \\
\hline AAAAAG & 5863 & AAAAAG & 5863 & EEDEEE & 3959 & GSGSGS & 3240 \\
\hline PSSSSS & 5813 & PSSSSS & 5813 & SSSSTS & 3953 & DEDEDE & 3127 \\
\hline NNNNNS & 5811 & NNNNNS & 5811 & GGGGSG & 3934 & EDEDED & 3045 \\
\hline QQQQQH & 5798 & QQQQQH & 5798 & AAVAAA & 3768 & RSRSRS & 2983 \\
\hline SSSSST & 5780 & SSSSST & 5780 & AAAVAA & 3758 & DDDDEE & 2953 \\
\hline DDDDDE & 5611 & DDDDDE & 5611 & GSGGGG & 3690 & EEEDDD & 2845 \\
\hline SNNNNN & 5585 & SNNNNN & 5585 & SSTSSS & 3660 & DDDEEE & 2822 \\
\hline ASSSSS & 5581 & ASSSSS & 5581 & SSSTSS & 3652 & RGRGRG & 2817 \\
\hline SAAAAA & 5405 & SAAAAA & 5405 & EEEDEE & 3627 & EEDDDD & 2754 \\
\hline АРPPPP & 5325 & АРPРPP & 5325 & AAAAVA & 3616 & AAAAGG & 2743 \\
\hline AAAAAS & 5322 & AAAAAS & 5322 & GGGSGG & 3556 & EDEDEE & 2651 \\
\hline AAAAAV & 5277 & AAAAAV & 5277 & SPSSSS & 3459 & DDEDED & 2570 \\
\hline GGGGGS & 5118 & GGGGGS & 5118 & NNSNNN & 3429 & RGGRGG & 2537 \\
\hline GGGGGA & 4862 & GGGGGA & 4862 & NNNSNN & 3418 & DEDEDD & 2489 \\
\hline PQQQQQ & 4819 & PQQQQQ & 4819 & AVAAAA & 3391 & SSSSTT & 2448 \\
\hline
\end{tabular}

different proteomes. The correlation coefficient $(r)$ was calculated using the equation

$$
r=\frac{(1 / n) \sum_{i=1}^{n}\left(x_{i}-\bar{x}\right)\left(y_{i}-\bar{y}\right)}{S_{x} S_{y}},
$$

where $S_{x}$ and $S_{y}$ are the standard deviations for variables $x$ and $y$.

For 20 homorepeats, the standard error in determining the correlation coefficient is less than $1 / \sqrt{20-2} \cong$ 0.24 . The standard error of correlation coefficient is $\mathrm{se}_{r}=$ $\sqrt{\left(1-r^{2}\right) /(n-2)}$ where $n$ is the number of points; for 109 disordered patterns it is less than $1 / \sqrt{109-2} \cong 0.1$, and for 11780 patterns it is less than 0.01 . Therefore, in Tables 3-7 the correlation coefficients range as follows: less than 0.5 , from 0.5 to 0.75 , and larger than 0.75 .

\section{Results and Discussion}

3.1. Occurrences of Motifs in 122 Proteomes. We constructed the library of all possible motifs consisting of the two amino acids, with the assumption that each amino acid could be at any position and at any ratio and that such a motif was six amino acid residues long. There were 11780 such motifs in total. The obtained motifs were divided into three groups (see Section 2). The numbers of motifs in the first, second, and third groups were 760 (6\%), $1520(13 \%)$, and 9500 (81\%), respectively. We estimated the occurrences of these motifs in 122 proteomes.

The most often occurrences of simple motifs for 122 proteomes from the three groups are presented in Table 1. Among the motifs from the first group, the leaders from the human proteome were EEEEED (422 times), DEEEEE (370), LPPPPP (327), APPPPP (264), PLLLLL (251), and PPPPPL (216). It should be noted that such motifs as LPPPPP, PLLLLL, and PPPPPL are not leaders among the occurrences of 122 proteomes (see Table 1). Among the motifs in which one amino acid occurred once and only inside the motif, the leaders from the human proteome were EEEEDE (288), EDEEEE (279), EEDEEE (248), EEEDEE (250), PLPPPP (239), and PPPPLP (207). Among the leaders in which the two amino acids occurred were SGSGSG (135), EEEEDD (157), GPPGPP (162), and RSRSRS (153). The following rare motifs that appeared only in two proteins should be noted for the human proteome: FFFFFN, FFFFFP, CHHHHH, MVVVVV, IHHHHH, WKKKKK, NNNNNS, and IIIIIF from the first group; IIMIII, RRFRRR, YLYYYY, NNCNNN, HHTHHH, and DDQDDD from the second group; and CCCRRR, MMMGGG, TTTDDD, FFSFFS, FFPFFP, VVRVVR, QQKQQK, and DDHDDH from the third group. At the same time, the NNNNNS motif is among the leader motifs for 122 proteomes and it occurs 146 times in the Drosophila melanogaster proteome and 473 times in the Plasmodium falciparum proteome (Alveolata kingdom). An analogous situation is observed for SNNNNN. It does not occur in the human proteome and appears in 489 proteins for the Plasmodium falciparum proteome. PQQQQQ occurs 52 times in the human proteome and 413 times in the Dictyostelium discoideum proteome.

In frequently occurring motifs from the Drosophila melanogaster proteome, the leading amino acids were glutamine, alanine, and glycine. Among the motifs from the first group, the leaders were QQQQQH (470), HQQQQQ (410), LQQQQQ (359), QQQQQL (359), QQQQQP (276), 


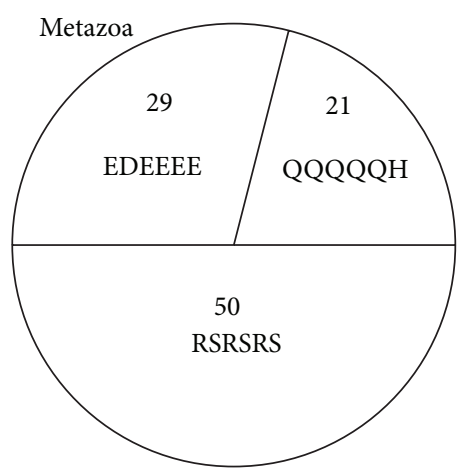

(a)

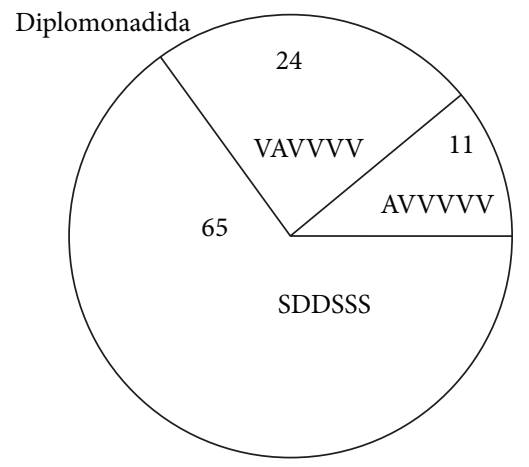

(c)

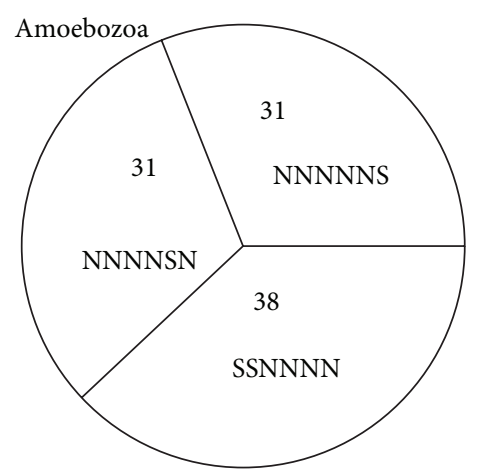

(b)

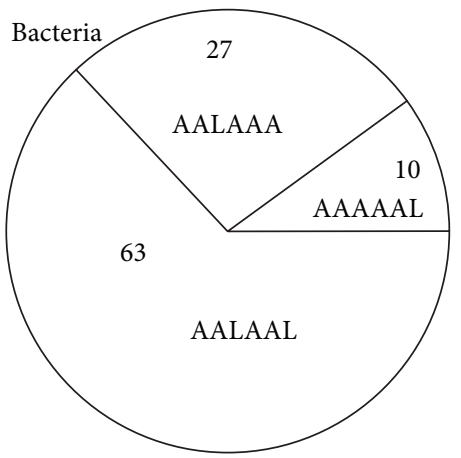

(d)

FIGURE 1: Statistics of occurrence of motifs, six residues long. Statistics of occurrence of motifs, six residues long, consisting of two amino acids in the three groups for 3 kingdoms of Eukaryota and for 5 phyla of Bacteria in percentage terms: (a) the Metazoa kingdom (17 proteomes), (b) the Amoebozoa kingdom (2 proteomes), (c) the Diplomonadida kingdom (3 proteomes), and (d) 26 bacterial proteomes. For each kingdom, the motif with the frequent occurrence in the group is presented.

PQQQQQ (260), QQQQQA (221), AQQQQQ (219), and SAAAAA (224). Among the motifs of the second group, the leaders were QQQQHQ (319), QQQHQQ (297), QQHQQQ (290), QHQQQQ (284), QQQQLQ (243), QLQQQQ (229), and QQLQQQ (218). Among the motifs of the third group, the leaders were GSGSGS (174), SGSGSG (157), HHQQQQ (163), QQQQHH (166), SSGGGG (110), and GGSGSG (105).

Out of 11780 motifs, 865 were not found in 122 proteomes.

We estimated the occurrence of the motifs from the three groups in 9 kingdoms of Eukaryota and 5 phyla of Bacteria (see Table 2). Interestingly, for all eukaryotic proteomes with the exception of the Amoebozoa and Diplomonadida kingdoms, the number of proteins containing at least one motif from the first group was about $20 \%$; in the case of motifs from the second and third groups, 30\% and 50\%, respectively (see Table 2). For bacterial proteomes this relationship is $10 \%, 27 \%$, and $63 \%$, respectively. One can see that proteomes from the Diplomonadida kingdom are more close to bacterial proteomes than to eukaryotic ones (see Figure 1). It should be noted that diplomonads are a group of flagellates, most of which are parasitic. At the same time, the proteomes from the Amoebozoa kingdom have different statistics: 31\%, $31 \%$, and 38\%, respectively. For the Metazoa, Amoebozoa, Diplomonadida, and Bacteria kingdoms, the motifs with the frequent occurrence in the groups are presented in Figure 1.

Among animal proteomes, one can see some deviation from the average values for Nematostella vectensis (class
TABLE 2: Occurrence of 11780 motifs from the three groups in 9 kingdoms of Eukaryota and for 5 phyla of Bacteria in percentage terms.

\begin{tabular}{|c|c|c|c|c|c|c|}
\hline Kingdom & \multicolumn{2}{|c|}{ First group } & $\begin{array}{l}<\mathrm{X}> \\
\mathrm{Sec}\end{array}$ & $\begin{array}{l}\text { Error } \\
\text { d group }\end{array}$ & $\begin{array}{l}<\mathrm{x}> \\
\text { Thir }\end{array}$ & $\begin{array}{l}\text { Error } \\
\text { group }\end{array}$ \\
\hline Metazoa (17) & 21 & 3 & 29 & 1 & 50 & 4 \\
\hline Viridiplantae (5) & 21 & 4 & 28 & 2 & 51 & 5 \\
\hline Stramenopiles (1) & 28 & - & 32 & - & 41 & - \\
\hline Choanoflagellida (1) & 18 & - & 27 & - & 55 & - \\
\hline Euglenozoa (4) & 22 & 3 & 29 & 2 & 49 & 4 \\
\hline Alveolata (6) & 23 & 4 & 29 & 1 & 48 & 5 \\
\hline Amoebozoa (2) & 31 & 1 & 31 & 0 & 38 & 2 \\
\hline Diplomonadida (3) & 11 & 1 & 24 & 1 & 65 & 2 \\
\hline Fungi (58) & 18 & 3 & 28 & 1 & 53 & 4 \\
\hline Bacteria (25) & 10 & 1 & 27 & 2 & 63 & 3 \\
\hline All 11780 motifs & 6 & 0 & 13 & 0 & 81 & 0 \\
\hline
\end{tabular}

Anthozoa, phylum Cnidaria): 14\%, 27\%, and 59\%, correspondingly. This is more close to the statistics for the bacterial proteomes. Another deviation from the average values is observed for phylum Arthropoda, especially for class Insecta (29\%, 30\%, and $41 \%$ for Anopheles darlingi and 26\%, 29\%, and 45\% for Anopheles gambiae). 


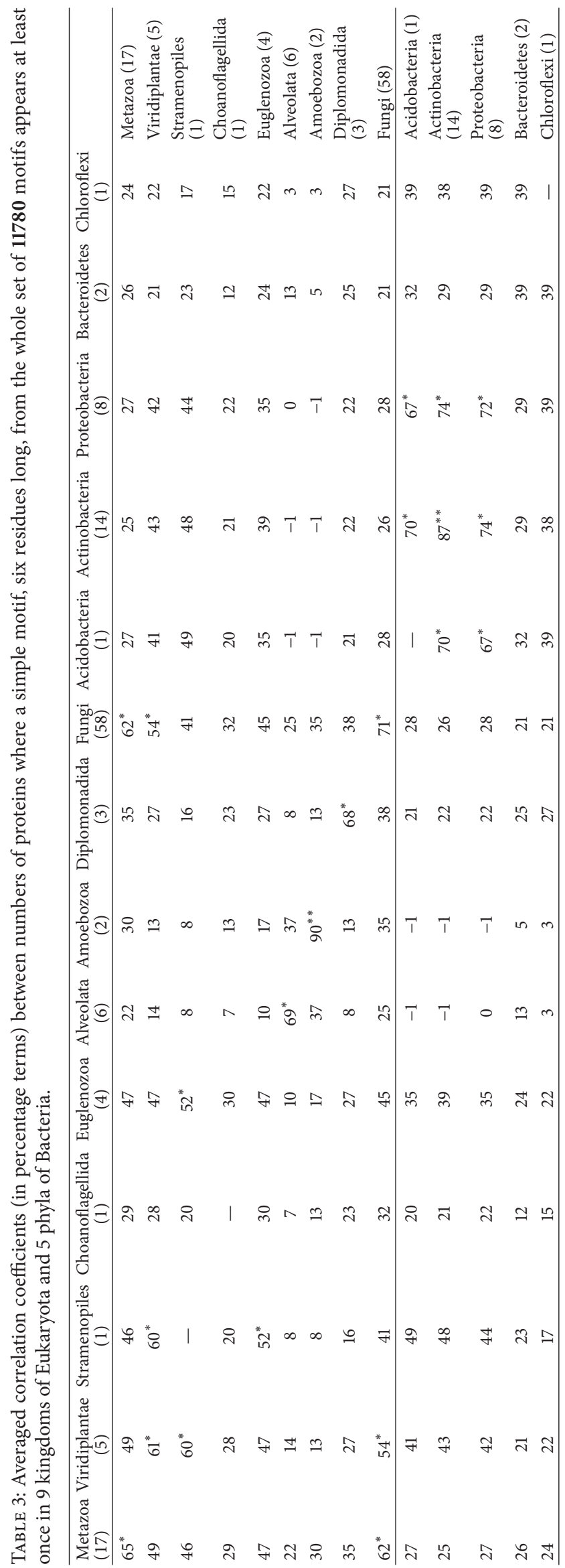




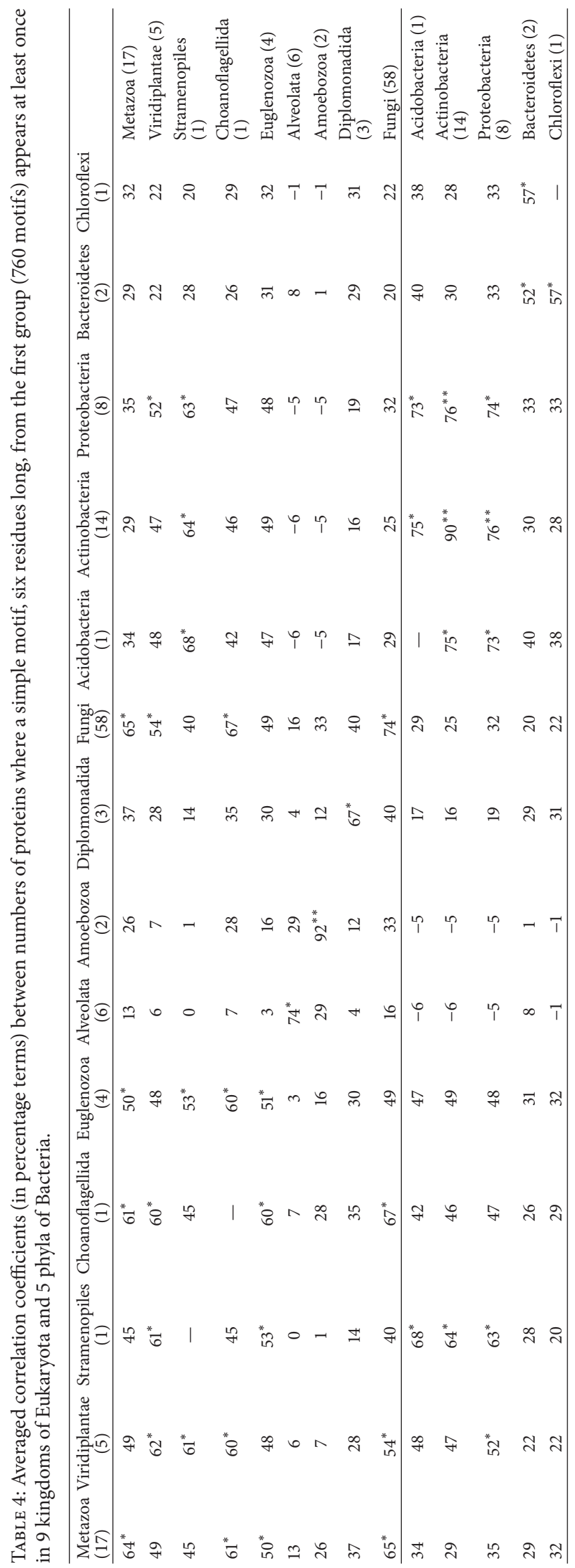




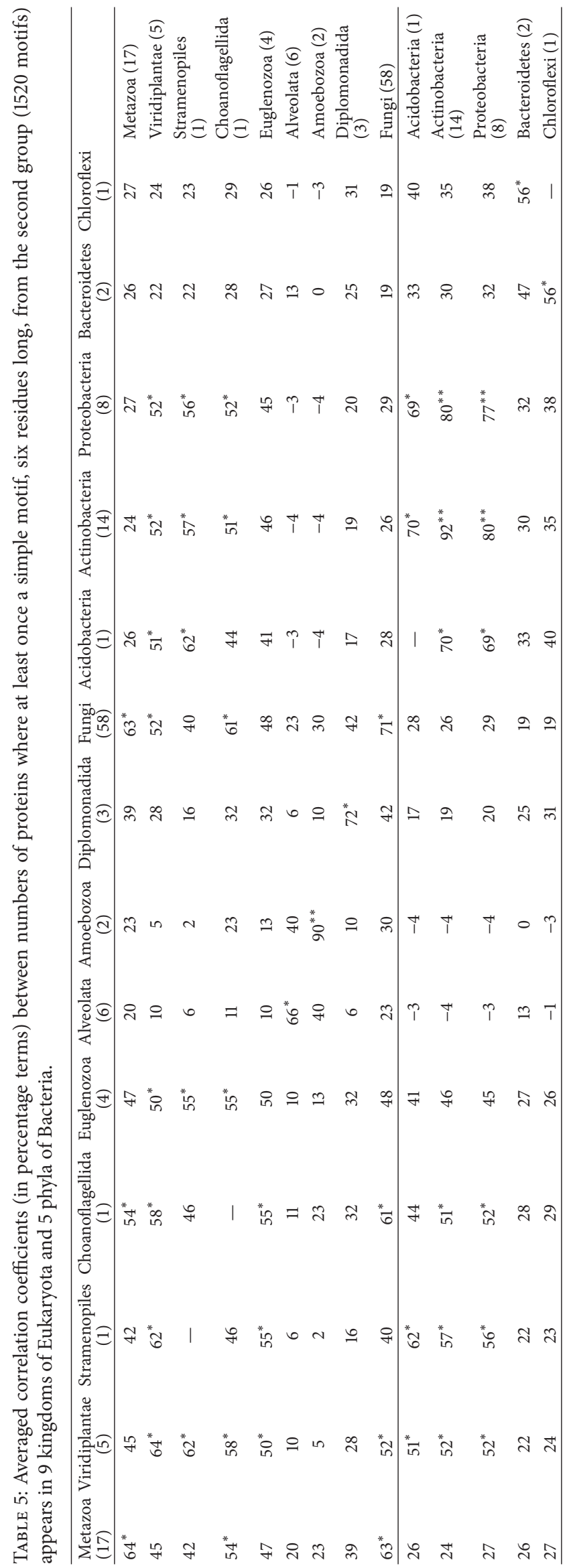




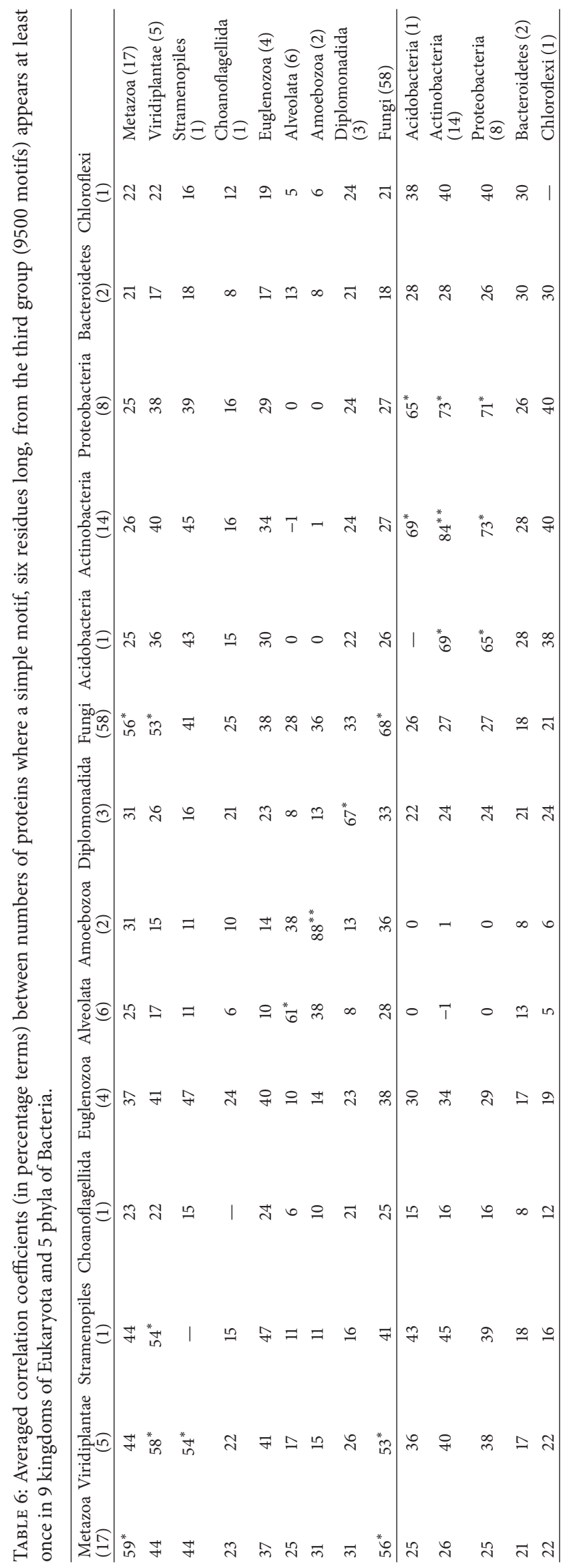




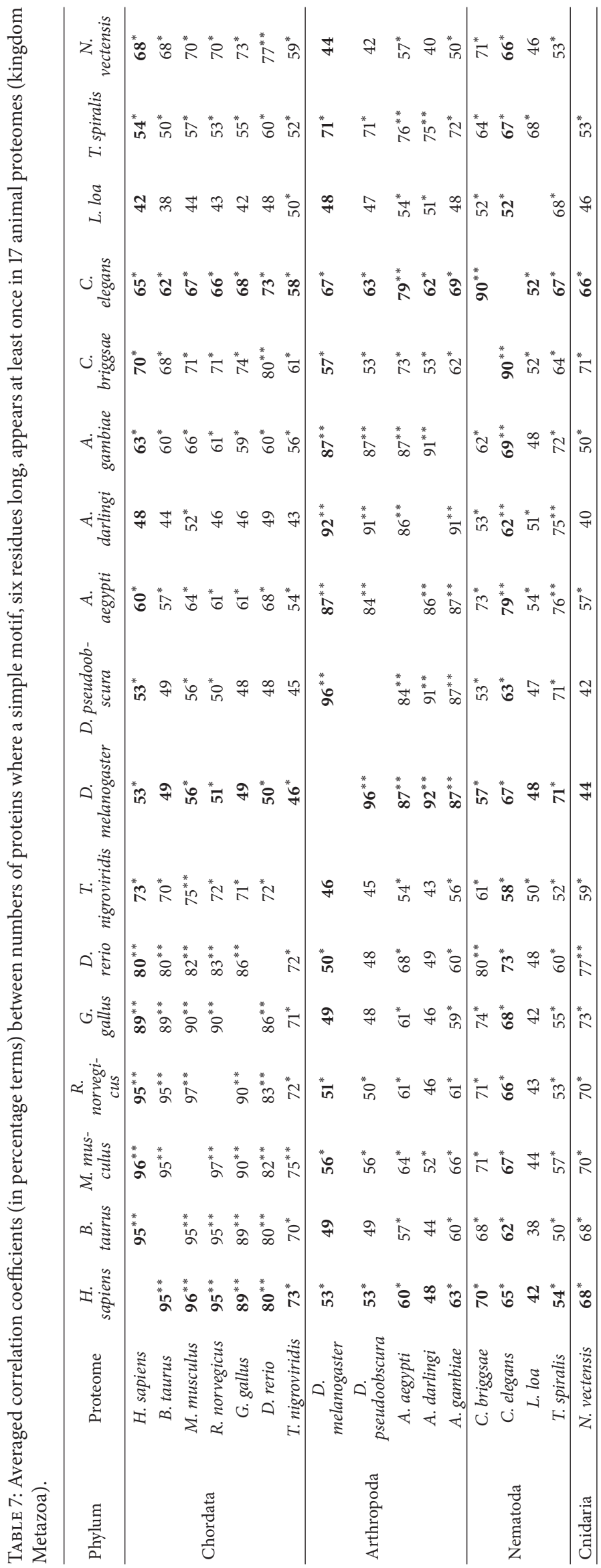


It should be also noted that the proteins bearing motifs from the third group occurred more frequently than the proteins with motifs from the two other groups only because the third group contained a significantly larger number of motifs (12.5 times as many as in the first group). It might be noted that motifs from the first groups are the simplest, being homorepeats with an adjacent amino acid. Motifs from the second group are homorepeats with an inclusion of the other amino acid. Meanwhile, members of the third group can hardly be derived from homorepeats. The most frequent motifs are the ones most closely resembling homorepeats, that is, the motifs from the first group, whereas the motifs from the second group occur somewhat more rarely, and the motifs not resembling homorepeats are the rarest of all. Each proteome contains its characteristic leading motifs, and it is apparent that the amino acids foremost among six amino acid repeats occur most often.

3.2. Construction of Matrices of Correlation Coefficients for Proteins Containing Simple Motifs in the Studied Proteomes. For each proteome, we calculated a set of 11780 values reflecting the number of proteins containing at least one simple motif, 6 residues long. Then considering all possible pairs of proteomes, the correlation coefficients between the 11780 values have been calculated which allowed us to construct a matrix of correlation coefficients (see Table 3). As a rule, the correlation coefficients are higher inside the studied kingdom than between them. A similar conclusion follows from considering the occurrence of motifs from the three groups (see Tables 4, 5, and 6). “**” in Tables 3-7 is used to show the correlation higher than $75 \%$, and " $*$ " is used to show the correlation from $50 \%$ to $75 \%$. Usually, the correlation coefficients are higher inside the considered kingdom than between them. The highest correlation is observed for the Amoebozoa kingdom in all cases (see Tables 3-6).

Most of the theories suggest that colonial naked choanoflagellate-like protists gave rise to first animals, while chitinous thecate choanoflagellate-like protists gave rise to first fungi $[30,31]$. In the case of occurrence of the motifs from the first and second groups, we obtained a high correlation between the Choanoflagellida and Fungi kingdoms (0.67 and 0.61) compared to between the Choanoflagellida and animals kingdoms (0.61 and 0.54) (see Tables 4-6).

We averaged the correlation coefficients over all proteomes from the studied kingdoms. The averaged correlation coefficient is low inside such a kingdom as Metazoa (see Table 3). We decided to analyze in more detail the proteomes from the Metazoa kingdom. If the correlation coefficients for animal proteomes only (see Table 7) are to be considered, four clusters can be selected with high correlation between the numbers of proteins where a simple motif, 6 residues long, appears at least once. The first cluster corresponds to the phylum Chordata (7 proteomes), the second to Arthropoda (5 proteomes), the third to Nematoda (4 proteomes), and the fourth to Cnidaria (only 1 proteome). Again one can see that the correlation coefficients are higher inside the considered phylum than between them.

In Table 7 one can see that the correlation coefficient between zebrafish, Danio rerio, and pufferfish, Tetraodon nigroviridis, is 0.72 , while on the other hand that between $D$. rerio and starlet sea anemone, Nematostella vectensis, is 0.77 and those between D. rerio and two nematodes, Caenorhabditis elegans and C. briggsae, are 0.73 and 0.80 , respectively. The correlation coefficients between T. nigroviridis and other vertebrates are $0.70-0.75$, while those between $D$. rerio and other vertebrates, except for T. nigroviridis, are 0.80-0.86. These values suggest that the pattern of six-residue-long motifs in $T$. nigroviridis has changed very rapidly after the separation of the lineages of pufferfish (belongs to a family of primarily marine and estuarine fish) and zebrafish (a tropical freshwater fish). This fact is not surprising in light of the last data, that horses were evolutionarily closest to Brandt's bats (Myotis brandtii); their divergence occurred about 81.7 million years ago, which is close to the time of the adaptive radiation of the class Mammalia [32].

In the case of the occurrence of simple motifs (all 11780 and 9500 for the third group), there is no high correlation (larger than 0.5 ) between eukaryotic and bacterial proteomes. Among the correlation coefficients for eukaryotic proteomes, there is a high correlation between the animal and Fungi kingdoms (0.62) compared to between the fungi and plants (0.54). This is valid also in the case of consideration of the correlation coefficients for the occurrence of the motifs from the three groups separately (see Tables 4-6). Moreover, this result agrees with the results obtained by us after analysis of loops in elongation factors EF1A using the novel informative characteristic called the "loops" method [20]. The method is based on the ability of amino acid sequences to form flexible loops in protein structure. Each kingdom displayed variations in the number of loops and their location within the three EF1A domains. It has been found that animals and fungi are sibling kingdoms [20].

\section{Conclusions}

One can see that some simple motifs have been maintained throughout evolution and that in the studied 122 eukaryotic and bacterial proteomes the most frequent motifs are specific for each proteome. The ratio between occurrences of the simple motifs from the three groups is practically the same for the eukaryotic proteomes. The other relationship between occurrences of the motifs is observed for the bacterial proteomes. The question about specificity of these motifs is more important for biological functioning. Our study provides support that animals and fungi are sibling kingdoms.

\section{Conflict of Interests}

The authors declare that there is no conflict of interests regarding the publication of this paper.

\section{Acknowledgments}

This study was supported by the Russian Science Foundation (Grant no. 14-14-00536) to O. V. Galzitskaya and by the Russian Academy of Sciences, Molecular and Cell Biology Program (Grant 01201353567) to M. Yu. Lobanov. 


\section{References}

[1] C. R. Woese and G. E. Fox, "Phylogenetic structure of the prokaryotic domain: the primary kingdoms," Proceedings of the National Academy of Sciences of the United States of America, vol. 74, no. 11, pp. 5088-5090, 1977.

[2] C. R. Woese, O. Kandler, and M. L. Wheelis, "Towards a natural system of organisms: proposal for the domains Archaea, Bacteria, and Eucarya," Proceedings of the National Academy of Sciences of the United States of America, vol. 87, no. 12, pp. 45764579, 1990.

[3] N. Iwabe, K. Kuma, M. Hasegawa, S. Osawa, and T. Miyata, "Evolutionary relationship of archaebacteria, eubacteria, and eukaryotes inferred from phylogenetic trees of duplicated genes," Proceedings of the National Academy of Sciences of the United States of America, vol. 86, no. 23, pp. 9355-9359, 1989.

[4] R. S. Gupta and B. Singh, "Cloning of the HSP70 gene from Halobacterium marismortui: relatedness of archaebacterial HSP70 to its eubacterial homologs and a model for the evolution of the HSP70 gene," Journal of Bacteriology, vol. 174, no. 14, pp. 4594-4605, 1992.

[5] N. Saitou and M. Nei, "The neighbor-joining method: a new method for reconstructing phylogenetic trees," Molecular Biology and Evolution, vol. 4, pp. 406-425, 1987.

[6] W. M. Fitch, "Toward defining the course of evolution: minimum change for a specified tree topology," Systematic Zoology, vol. 20, no. 4, pp. 406-416, 1971.

[7] J. W. Pratt, "F. Y. Edgeworth and R. A. Fisher on the efficiency of maximum likelihood estimation," The Annals of Statistics, vol. 4, no. 3, pp. 501-514, 1976.

[8] R. A. Lippert, H. Huang, and M. S. Waterman, "Distributional regimes for the number of $K$-word matches between two random sequences," Proceedings of the National Academy of Sciences of the United States of America, vol. 99, no. 22, pp. 13980-13989, 2002.

[9] G. Reinert, D. Chew, F. Sun, and M. S. Waterman, "Alignmentfree sequence comparison I: statistics and power," Journal of Computational Biology, vol. 16, no. 12, pp. 1615-1634, 2009.

[10] L. Wan, G. Reinert, F. Sun, and M. S. Waterman, "Alignmentfree sequence comparison (II): theoretical power of comparison statistics," Journal of Computational Biology, vol. 17, no. 11, pp. 1467-1490, 2010.

[11] X. Liu, L. Wan, J. Li, G. Reinert, M. S. Waterman, and F. Sun, "New powerful statistics for alignment-free sequence comparison under a pattern transfer model," Journal of Theoretical Biology, vol. 284, pp. 106-116, 2011.

[12] J. M. Comeron, R. Ratnappan, and S. Bailin, "The many landscapes of recombination in Drosophila melanogaster," PLoS Genetics, vol. 8, no. 10, Article ID e1002905, 2012.

[13] M. Gouy and W. H. Li, "Molecular phylogeny of the kingdoms animalia, plantae, and fungi," Molecular Biology and Evolution, vol. 6, no. 2, pp. 109-122, 1989.

[14] G. K. Philip, C. J. Creevey, and J. O. McInerney, "The Opisthokonta and the ecdysozoa may not be clades: stronger support for the grouping of plant and animal than for animal and fungi and stronger support for the Coelomata than ecdysozoa," Molecular Biology and Evolution, vol. 22, no. 5, pp. 1175-1184, 2005.

[15] S. Yang, R. F. Doolittle, and P. E. Bourne, "Phylogeny determined by protein domain content," Proceedings of the National Academy of Sciences of the United States of America, vol. 102, no. 2, pp. 373-378, 2005.
[16] A. Löytynoja and M. C. Milinkovitch, "Molecular phylogenetic analyses of the mitochondrial ADP-ATP carriers: the plantae/fungi/metazoa trichotomy revisited," Proceedings of the National Academy of Sciences of the United States of America, vol. 98, no. 18, pp. 10202-10207, 2001.

[17] P. O. Wainright, G. Hinkle, M. L. Sogin, and S. K. Stickel, "Monophyletic origins of the metazoa: an evolutionary link with fungi," Science, vol. 260, no. 5106, pp. 340-342, 1993.

[18] S. L. Baldauf and J. D. Palmer, "Animals and fungi are each other's closest relatives: congruent evidence from multiple proteins," Proceedings of the National Academy of Sciences of the United States of America, vol. 90, no. 24, pp. 11558-11562, 1993.

[19] N. Nikoh, N. Hayase, N. Iwabe, K.-I. Kuma, and T. Miyata, "Phylogenetic relationship of the Kingdoms Animalia, Plantae, and Fungi, inferred from 23 different protein species," Molecular Biology and Evolution, vol. 11, no. 5, pp. 762-768, 1994.

[20] V. G. Oxana, I. D. Eugeniya, and N. S. Igor, "Phylogenetic analysis of the loops in elongation factors EF1A: stronger support for the grouping of animal and fungi," Journal of Computer Science and System Biology, vol. 1, pp. 073-080, 2008.

[21] M. Y. Lobanov and O. V. Galzitskaya, "Occurrence of disordered patterns and homorepeats in eukaryotic and bacterial proteomes," Molecular BioSystems, vol. 8, no. 1, pp. 327-337, 2012.

[22] M. Y. Lobanov and O. V. Galzitskaya, "Disordered patterns in clustered protein data bank and in eukaryotic and bacterial proteomes," PLoS ONE, vol. 6, no. 11, Article ID e27142, 2011.

[23] M. Y. Lobanov, E. I. Furletova, N. S. Bogatyreva, M. A. Roytberg, and O. V. Galzitskaya, "Library of disordered patterns in 3D protein structures," PLoS Computational Biology, vol. 6, no. 10, Article ID 1000958, 2010.

[24] M. Y. Lobanov, I. V. Sokolovskiy, and O. V. Galzitskaya, "IsUnstruct: prediction of the residue status to be ordered or disordered in the protein chain by a method based on the Ising model," Journal of Biomolecular Structure and Dynamics, vol. 31, no. 10, pp. 1034-1043, 2013.

[25] E. Salichs, A. Ledda, L. Mularoni, M. M. Albà, and S. de la Luna, "Genome-Wide analysis of histidine repeats reveals their role in the localization of human proteins to the nuclear speckles compartment," PLoS Genetics, vol. 5, no. 3, Article ID e1000397, 2009.

[26] J. P. Zbilut, G. H. Chua, A. Krishnan, C. Bossa, M. Colafranceschi, and A. Giuliani, "Entropic criteria for protein folding derived from recurrences: six residues patch as the basic protein word," FEBS Letters, vol. 580, no. 20, pp. 4861-4864, 2006.

[27] O. V. Galzitskaya, "Search for folding initiation sites from amino acid sequence," Journal of Bioinformatics and Computational Biology, vol. 6, no. 4, pp. 681-691, 2008.

[28] G. V. Nikiforovich and C. Frieden, "The search for local nativelike nucleation centers in the unfolded state of $\beta$-sheet proteins," Proceedings of the National Academy of Sciences of the United States of America, vol. 99, no. 16, pp. 10388-10393, 2002.

[29] M. Y. Lobanov, N. S. Bogatyreva, and O. V. Galzitskaya, "Occurrence of six-amino-acid motifs in three eukaryotic proteomes," Molecular Biology, vol. 46, no. 1, pp. 168-173, 2012.

[30] T. Cavalier-Smith, "The origin of fungi and pseudogungi," in Evolutionary Biology of Fungi, A. D. M. Rayner, C. M. Braisier, and D. Moore, Eds., pp. 339-353, Cambridge University Press, Cambridge, UK, 1987.

[31] K. Buck, "Phylum zoomastigina, class choanomastigotes (choanoflagellates)," in Handbook of Protista, L. Margulis, J. O. 
Corliss, M. Melkonian, and D. J. Chapman, Eds., pp. 194-199, Jones and Bartlett Publishers, 1990.

[32] I. Seim, X. Fang, Z. Xiong et al., "Genome analysis reveals insights into physiology and longevity of the Brandt's bat Myotis brandtii," Nature Communications, vol. 4, article 2212, 2013. 

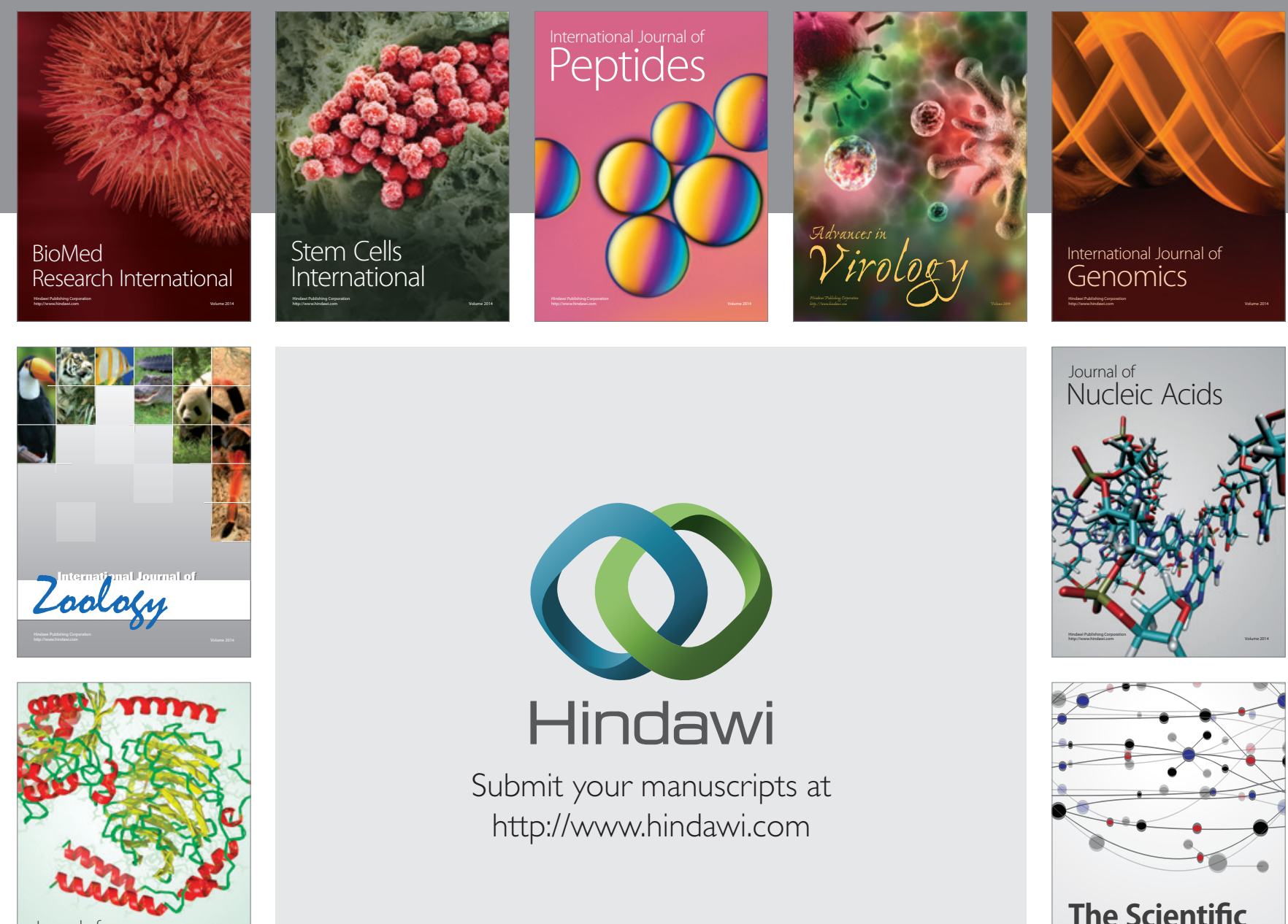

Submit your manuscripts at

http://www.hindawi.com

Journal of
Signal Transduction
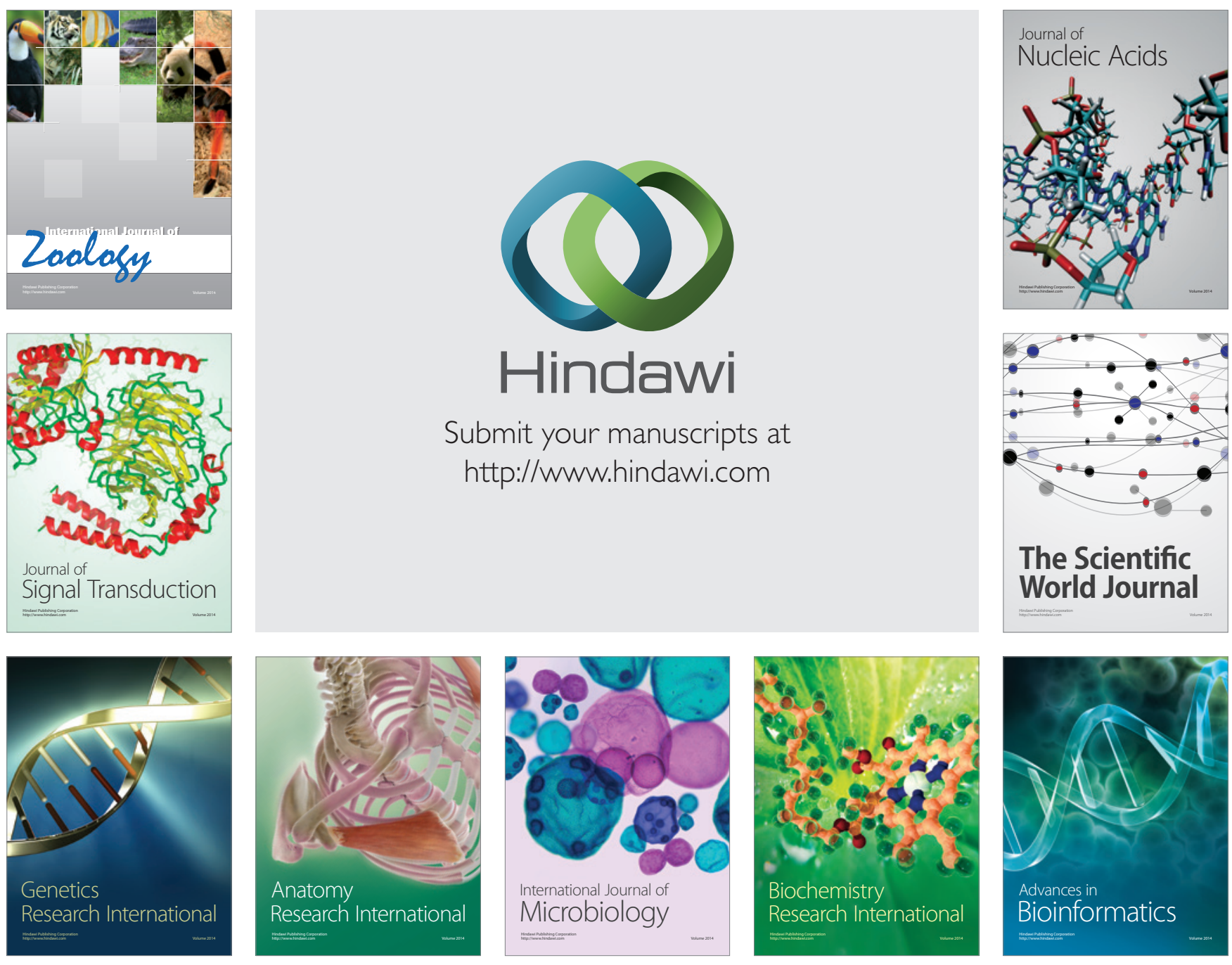

The Scientific World Journal
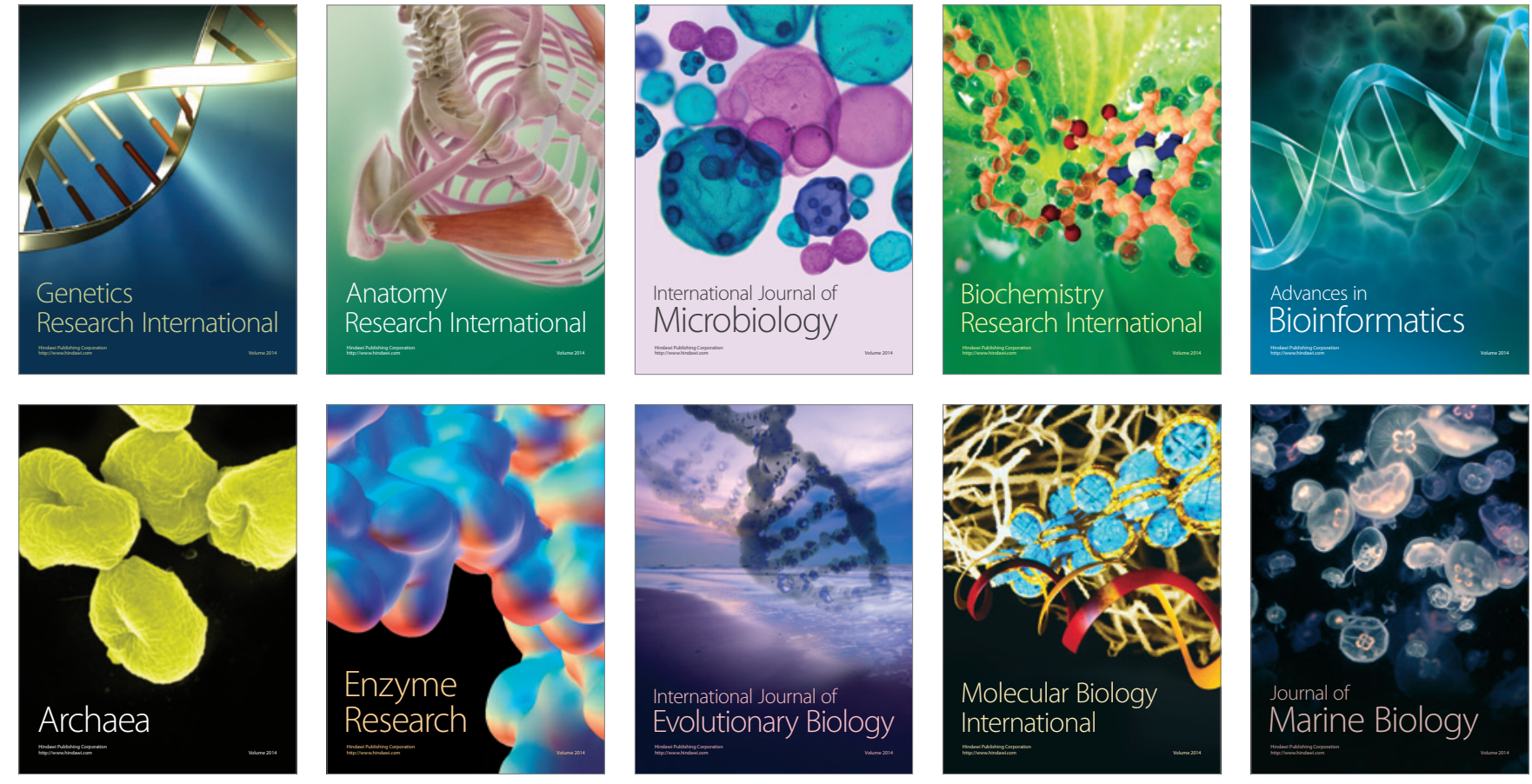